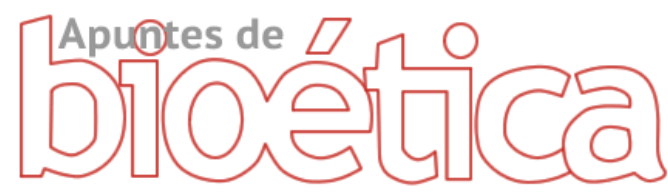

https://doi.org/10.35383/apuntes.v2i2.292

\title{
De la familia a la Biofamilia
}

\author{
Alicia Ruth Jiménez Carbajal ${ }^{1}$
}

\section{INFORMACIÓN DEL ARTÍCULO RESUMEN}

\section{Historia del artículo:}

Recibido el 27 de setiembre de 2019

Aceptado el 26 de diciembre de 2019

\section{Palabras claves: \\ Ética \\ Biofamilia \\ Humanización \\ Ideología}

El término Biofamilia se puede considerar un neologismo necesario para los estudios e investigaciones acerca de la familia. Ésta, con sus costumbres y comportamientos también adquiere interés desde la ética. De la ética aplicada a la familia surge el término biofamilia, el cual hace referencia al primer grupo de vida, donde la persona se humaniza. La globalización ha permitido que ingresen ideologías que le quitan a la familia natural el lugar que le corresponde, las políticas públicas deben considerar la estructura y funcionamiento de la biofamilia como fundamento para el desarrollo de la sociedad. La relativización del concepto de familia por parte de los organismos supranacionales ha contribuido a aumentar la confusión. El desarrollo del concepto de biofamilia, en los ambientes académicos y de investigación permitirá no desvirtuar el concepto de familia

\section{From the family to the Biofamily}

\section{ABSTRACT}

\section{Keywords:}

Ethics

Biofamily

Humanization

Ideology
The term Biofamily can be considered a necessary neologism for studies and research about the family. This, with its customs and behaviors also acquires interest from ethics. From the ethics applied to the family the term biofamily arises, which refers to the first life group, where the person becomes human. Globalization has allowed the entry of ideologies that take the natural family from its rightful place; public policies must consider the structure and functioning of the biofamily as the foundation for the development of society. The relativization of the family concept by supranational organizations has contributed to increased confusion. The development of the concept of biofamily, in academic and research environments will allow not to distort the concept of family.

${ }^{1}$ Estudios concluidos en Persona, Matrimonio y Familia, Licenciada en Estadística. Realiza consultoría y asesoría en área de matrimonio, familia y noviazgo. Lima, Perú Email: alicjimenez@yahoo.com ORCID: https://orcid.org/0000-0002-8221-7443 


\section{Introducción}

La familia es la célula básica de la sociedad y por su importancia debería tener prioridad en las políticas de gobierno de todo país. Por su importancia es objeto de múltiples estudios y abordada desde diferentes disciplinas debido a la relación que tiene con el despliegue de las potencialidades del ser humano.

La ética aplicada a la familia tiene mucho que aportar sobre el tema. Para este efecto ha surgido el neologismo biofamilia que viene siendo utilizado en los ambientes académicos.

Se partirá analizando la importancia de la ética en el siglo XXI, y cómo la ética aplicada da lugar al concepto de biofamilia $a_{i=}^{-}$evidenciando la necesidad del enfoque de biofamilia para enfrentar los nuevos retos de la familia en la sociedad.

Por lo tanto el objetivo de este artículo es analizar el significado del término, en los estudios sobre la familia de tal modo que se pueda hablar de un enfoque de biofamilia para proteger su esencia conceptual, la cual se ha visto alterada por los fenómenos sociales y las ideologías de la época.

\section{Metodología}

Esta ha sido de tipo cualitativa descriptiva, enfoque utilizado principalmente en las ciencias sociales. Se buscó identificar la naturaleza del problema y describir los diferentes aspectos que corresponden al contenido del tema en estudio. Se realizó una búsqueda bibliográfica exhaustiva relacionada al tema de biofamilia y afines.

Los criterios éticos utilizados corresponden a la filosofía personalista: pone el énfasis en la persona, considera al hombre como un ser relacional, esencialmente social y comunitario, un ser libre, trascendente y con un valor en sí mismo que le impide convertirse en un objeto como tal.

\section{Resultados, análisis y discusión}

\section{Ética, ¿valoración de comportamientos en el siglo XXI?}

La ética como disciplina filosófica toma como objeto de estudio a la moral y se convierte en la teoría del comportamiento moral, fundamenta, explica y porque no decirlo, también critica las prácticas y normas morales.

Nuestra sociedad contemporánea ha sido denominada sociedad posmoralista ${ }^{2}$ es decir una sociedad más allá del bien o del mal, primando la neutralidad y la objetividad, dejando de lado la moral.

Aparentemente la ética no tendría entonces lugar en una sociedad donde prima la objetividad; y los valores, las exigencias morales y el altruismo se han debilitado y ya no tienen mucho éxito. Paradójicamente esta misma sociedad contemporánea promueve la ética en diversos ambientes laborales, medios de comunicación e inclusive el académico. Lipovetsky (1994) responde a esta paradoja estableciendo que "la sociedad posmoralista repudia la retórica del deber austero, rechaza la entrega personal en bien del otro, pero corona los derechos subjetivos relacionándolos con la moral". Es aquí donde la ética recobra fuerza, pero en el marco de una moral indolora. Los individuos están deseosos de reglas justas, pero no de renuncia a sí mismo; apelan a la responsabilidad no a la obligación. Fue Dulkheim (1893/2007) quien formula que "el paso a la modernidad desestabiliza y genera desconcierto al punto de poner a la sociedad en un estado de

\footnotetext{
2 La época posmoralista es aquella en la cual la cultura cotidiana está dominada no solo por los objetos y el psicologismo sino también por la información: la lógica de la relegación de la retórica del deber es hija del consumo y la comunicación de masas. (Lipovetzky Gilles, 2000-, pag.53)
} 
anomia, es decir un estado de no integración o de desorganización social". La sociedad moderna carece de reglas que la cohesionen y valores compartidos. Por ejemplo la tolerancia se presenta actualmente como un valor compartido pero fomenta más los derechos subjetivos que los derechos para el bien común.

El individuo que pertenece a una sociedad anomia, es decir ${ }_{L}$ el individuo anómico, es un "individuo que se ha hecho espiritualmente estéril, responsable ante sí mismo y ante nadie más". (Camps, 1991, pág. 118) Es decir, una persona centrada en su propio yo.

Dentro del comportamiento humano es natural la búsqueda de reglas que lo orienten; a decir de la misma autora: "el comportamiento humano es comportamiento conforme a unas reglas, la crítica de las reglas vigentes no puede hacerse sino desde la búsqueda de otras normas". Esta ambivalencia, que implica rechazar reglas y al mismo tiempo necesitarlas, es consecuencia del pluralismo ideológico, y la cantidad de propuestas que se presentan como sustituto de la realidad objetiva.

Podríamos inferir que la ética no desaparecerá porque la cantidad de propuestas hace que la sociedad se vuelva cada vez más compleja, multiplicando de este modo los temas morales a tratar y en consecuencia se podrá aplicar a los diversos temas controversiales derivados de la ciencia, de las leyes y todos los problemas comunes a la humanidad en general. Las éticas profesionales o deontologías forman parte de la ética aplicada.

Dentro del campo de la ética aplicada se van acuñando términos. Uno de los más conocidos y utilizados es la BIOÉTICA que significa valoración de los comportamientos frente a la vida; vida y muerte en manos del ser humano. La bioética no se limita a la ética médica; por el contrario, la incluye", (Brunet, 2006, pág. 208).

\section{De la familia a la biofamilia}

La familia está compuesta por seres humanos, cada uno "desde que tiene por cuerpo una célula hasta el final natural del ciclo vital, en coherencia con que todo ser vivo pertenece a su especie durante su ciclo vital completo y que la igual dignidad en cuanto humanos se posee toda la vida". (Posada Gonzáles, julio-diciembre de 2017)

Al interior de la familia esta realidad la vive cada uno de sus miembros de tal modo que su proceso de humanización se vive, en primer lugar, en este recinto particular y privado, con características propias. Cada miembro, en su realidad corpóreo espiritual, creará y desarrollará vínculos al interior de esta familia y también aportará a lo largo del tiempo las experiencias de su contacto con el medio que lo rodea, los cuales llevará consigo cuando le toque formar su propia familia.

El estudio sobre el ser humano no se puede realizar únicamente desde su dimensión individual, es por esto que la persona debe ser explicada desde su contexto familiar. La familia como institución (natural y social) compleja amerita un estudio profundo y científico. La familia como ciencia tiene trayectoria en países como Estados Unidos, Canadá, Australia, Reino Unido, India. En América del Sur, en Colombia se está impulsando la discusión enfocada en la ciencia de la familia y su disciplina, la "familiología" (López Montaño, enero-julio del 2014, pág.66).

El estudioso de familia que le dio el mayor impulso para el reconocimiento de la ciencia de familia como una nueva disciplina fue Wesley Burr. Su mirada académica sobre la teoría de 
familia y su papel como presidente del NCFR: Consejo Nacional de Relaciones Familiares ${ }^{3}$, ayudó a conceptualizar la necesidad de tener ese reconocimiento. Burr argumentó que el estudio de la familia había dejado atrás sus raíces interdisciplinarias para convertirse en una nueva disciplina. (López Montaño, enero-julio del 2014, pág.72)

La familia con sus costumbres y comportamientos también adquiere interés desde la ética. De la ética aplicada a la familia surge el término biofamilia.

Gamboa (2014) justifica la biofamilia señalando que:

\section{La Biofamilia es la parte de la} Bioética que trata sobre cada ser humano como miembro de la familia de sangre, de esta como tal, y las relaciones entre sus miembros $y$ con el entorno social $y$ ambiental, teniendo en cuenta las diferencias específicas entre esta forma de familia como célula de la sociedad humana y otras como la que se acrecienta por la adopción y demás vínculos éticos.

Otra definición al respecto corresponde a la doctora Nubia Posada Gonzáles (2015), representante del FUCEB (Fundación Colombiana de Ética y Bioética):

La vida abarca al ser personal corpóreo entero y su pleno desarrollo humano integral, incluyente y sostenible, que es la meta de cada uno, la familia -a esto se dedica la parte de la Bioética denominada "Biofamilia"

Este prefijo "bio", con todo lo que él implica, aplicado al concepto de familia permite

${ }^{3}$ Organismo que agrupa, desde 1930, a profesionales de Familia de Estados Unidos, Canadá, Australia y algunos países de Europa. Wesley R. Burr fue presidente de este organización en el periodo 1981-82 acercarse a su realidad fundante, ontológica. Es como ponerse acorde con los tiempos con un despliegue de permanencia en donde la esencia de la familia será la misma en cualquier época.

\section{La biofamilia en la sociedad}

Al utilizar el término biofamilia se va a hacer referencia a la familia no solo como grupo de personas sino como primer grupo de vida, haciendo referencia no solo al ambiente donde el individuo mora sino, también ${ }_{\perp}$ al ambiente donde tiene sueños, hunde sus fracasos, recibe amor, indiferencia o tal vez rechazo; al lugar donde se generan los primeros vínculos, lugar donde el ser humano vive su existencia como tal. Sin temor a ser redundante, en palabras de Raquel Peña ${ }_{L}$ (2019) "el primer grupo de vida".

Este nuevo objeto de estudio, la biofamilia, recibe la influencia del entorno donde se desarrolla. Recibe la influencia de los fenómenos sociales (violencia, aborto, ideologías, entre otros) a los que está expuesta la sociedad y no siempre posee los saberes necesarios para enfrentarla. Un estudio muy interesante al respecto, de la ya citada Raquel Peña, responde precisamente a la pregunta: ¿Qué saberes poseen las familias en relación a los fenómenos sociales que asfixian a la sociedad actual y qué rol puede cumplir la educación en función de aminorar los flagelos que la afectan día a día? La autora demuestra- desde la visión familiar- que la educación comunitaria, la cual se presenta como un abanico de oportunidades para despertar consciencia en el colectivo y hacer frente a los fenómenos sociales desde una visión transeducativa, definitivamente se encuentra vinculada con la ética de la vida. Los componentes estructurales de su estudio son: biofamilia, bionova ${ }^{4}$ y bioeducación ${ }^{5}$ social. Se

${ }^{4}$ La misma autora define BIONOVA: componente imbricado con la unidad de análisis de los fenómenos sociales, en su propio nombre se fusionan en forma dual la vida y la destrucción. 
concluye que la educación comunitaria permite la transformación del individuo para un comportamiento saludable en la sociedad.

La familia se ve influenciada por los fenómenos sociales y urge la necesidad de un término que la proteja en su esencia: la biofamilia. Ya algunos investigadores hablan del enfoque biofamiliar ${ }^{6}$.

\section{Enfoque de biofamlia}

Para Bunge (1988) un enfoque es una manera de ver las cosas o las ideas $y$, en consecuencia, también de tratar los problemas relativos a ellas.

Desde no hace mucho tiempo- ha surgido el neologismo BIOFAMILIA, entendiéndose como ética aplicada a la familia. No estamos frente a un término más, si bien es un neologismo, surge sobre todo como una necesidad más que como la definición de una estructura. (Gamboa 2014, julio-diciembre del 2014).

La familia viene siendo objeto de estudio por la importancia que tiene como célula básica de la sociedad. El término biofamilia, como se ha delineado en el punto anterior, considera a la familia no como un ente estático sino que toma en cuenta tanto al ser humano que la_conforma, como su desarrollo humano e integral. Se podría decir la "vida" de la familia con su dinamismo de permanencia a través del tiempo. Entonces considerar un enfoque de biofamilia tanto para su análisis como para la elaboración de políticas públicas, permitiría también encontrar soluciones desde esta perspectiva humanista e integral. Sobre todas estas cuestiones, la Bioética

${ }^{5}$ La mencionada autora define BIOEDUCACIÓN: una educación que produce una transformación moral, ética y espiritual.

${ }^{6}$ Por ejemplo, la educación en sexualidad con enfoque de biofamilia. Estado del arte de documentos impresos de mediación pedagógica sobre sexualidad para profesores, niños y adolescentes, enviados por el Ministerio de Educación Nacional a instituciones de educación formal en Colombia (19902014) tiene algo que decir, por eso se justifica la emergencia de la Biofamilia. (Gamboa, juliodiciembre, 2014, pág. 104)

Colombia es uno de los países de Latino América que más aportes viene realizando en este tema. Vale la pena mencionar explícitamente de qué modo vienen trabajando el enfoque de biofamilia:

Secular: tiene en cuenta el reconocimiento de los aportes de todos, racionalmente sostenibles y argumentables. Se reconoce el derecho de cualquier miembro de la familia humana a la expresión coherente de sus convicciones y creencias, en el marco del respeto incondicional a cada uno de todos los seres humanos durante su ciclo vital completo. (Durand, 1992, pp. 23-28).

Global: en los pensamientos, palabras y actividades, se tiene en cuenta a cada ser humano entero, con una jerarquía ordenada y sustentada en argumentos razonables, según el grado de sus perfecciones y el sentido de su ser y de su existencia como totalidad en la unidad corpóreo-espiritual en que consiste su dotación natural, y en función de su pleno desarrollo integral, incluyente y sostenible, que reclama el cuidado de la naturaleza con enfoque transtemporal y transcultural. La Unesco señala: "Teniendo presente también que la identidad de una persona comprende dimensiones biológicas, psicológicas, sociales, culturales y espirituales" (Organización de las Naciones Unidas para la Educación, la Ciencia y la Cultura (Unesco), 2005).

Interdisciplinario: Potter, que durante más de tres décadas fue considerado el fundador de la bioética (Potter, Bioethics, science of survival, 1970, p. 127), 
afirmaba: "Bioética Puente es una expresión abreviada para la visión que cristalicé cuando acuñé el término en el año 1970. [...] Se utiliza la palabra puente ya que la bioética era vista como una nueva disciplina que forjaría una unión entre la ciencia y las humanidades, o con mayor precisión, un puente entre la ciencia biológica y la ética, por consiguiente: 'BIO- ETICA' [sic]. (Potter, 1998, p. 24).

Sistemático: requiere una organización desde la reflexión más rigurosa posible, para desarrollarse siguiendo un plan coherente con lo que se concluye como probablemente más acertado, según los contenidos sobre valores -bienes en cuanto reconocidos-principios referentes intelectuales que aseguran el pleno desarrollo humano coherente con los valores $y$, por eso, integral, incluyente $y$ sostenible - y normas -derechos $y$ deberes que promueven la gestión responsable de la libertad consigo mismo y con todos los demás.

Prospectivo: la bioética tiene en cuenta todo el pasado y aporta al presente con nuevas reflexiones que facilitan la vida actual y futura, a corto, mediano y largo plazo (Durand G, 1992, pp. 23-28). La responsabilidad reclama tener en cuenta no solo las decisiones cotidianas, sino también las consecuencias a largo plazo de las acciones que se recomendaron $o$ que se dejaron de considerar (Potter, 1998, p. 24).

Estas pautas de trabajo sobre el enfoque de biofamilia, corresponde a un artículo de investigación que lleva por título "Estado del arte de documentos impresos de mediación pedagógica sobre sexualidad, para profesores, niños y adolescentes, enviados por el Ministerio de Educación Nacional a instituciones de educación formal en Colombia (1990-2014)" con un equipo de investigación liderado por la doctora Nubia Posada. ${ }^{7}$ La sexualidad es un tema tan íntimo del núcleo familiar que difícilmente puede ser impuesto por políticas gubernamentales sino se trabaja desde un enfoque de biofamilia.

\section{Familia en los organismos supranacionales}

La era de la globalización como proceso de interacción e integración en el ámbito internacional no podía dejar fuera a la familia. La ONU en su preocupación por esta institución natural, en 1983, a través de la Comisión de Desarrollo Social, pide al secretario general (en ese entonces Javier Pérez de Cuellar) intensificar los esfuerzos por las necesidades y los problemas de la familia. En 1985, se incluye como tema de agenda. Producto de estas conversaciones, en 1994 se proclama "El año internacional de la familia" y se elige el 15 de mayo como fecha para su celebración.

En 1996, la ONU quiso centrar su atención en el papel que juega la familia en el desarrollo, así como, las tendencias recientes en la elaboración de políticas de familia. Pero los principios que se incluyeron en la proclamación del Año Internacional de la Familia, contenía un concepto diferente al manifestado en la Declaración Universal de los derechos humanos:

La familia es el elemento natural y fundamental de la sociedad y tiene

\footnotetext{
7 El Artículo de investigación derivado del proyecto: "Perfil crítico de documentos impresos de mediación pedagógica sobre sexualidad en educación formal en Colombia", estuvo asociado al grupo de investigación "Familia y sociedad", reconocido por Colciencias; su línea de investigación es "Sostenibilidad de la familia", en el grupo de la línea "Biofamilia de las primeras etapas de la vida. Bases del pleno desarrollo humano integral y sostenible. Fue llevado a cabo por cuatro grupos de investigadores (con la doctora Nubia Posada a la cabeza), elaboraron un estado del arte de materiales didácticos impresos sobre sexualidad, que fueron enviados por el Ministerio de Educación Nacional (MEN) a instituciones de educación formal en Colombia. Como resultado se sugiere la necesidad de elaborar nuevos materiales didácticos de mediación pedagógica sobre sexualidad, con referentes bioéticos específicos de biofamilia.
} 
derecho a la protección de la sociedad y del Estado. (Declaración Universal de Derechos Humanos. Artículo 16, numeral $3,1948)$

Las familias asumen diversas formas y funciones de un país a otro, $y$ dentro de cada sociedad nacional. Estas expresan la diversidad de las preferencias individuales $y$ las condiciones sociales. En consecuencia, el Año Internacional de la Familia abarca y aborda las necesidades de todas las familias. ${ }^{8}$ (Proclamación del Año Internacional de la Familia (1994)

Definitivamente se observa una completa diferencia en cuanto al concepto de familia. En la actualidad existen políticas originadas y auspiciadas por la ONU con propuestas negativas para la familia. Tal es el caso de los "Objetivos del Desarrollo del milenio" donde la familia no aparece entre ellos y en su lugar parece que se ha cedido el lugar a la promoción de la igualdad de género ${ }^{9}$

Existen planes organizados en detrimento de la familia natural, como lo describe Gabriel Kuby:

Desde la década de 1960, con la ayuda de las Naciones Unidas (ONU), la Unión Europea (UE) y los medios de comunicación, un poderoso lobby ha estado luchando para cambiar el sistema de valores. El objetivo es la libertad absoluta, sin ninguna restricción [...] Cuando la libertad se entiende de este modo, no hay "bien" ni "mal», ni ningún sistema de normas [...] El proceso es asombroso, porque este intento de crear un nuevo ser humano, y la consiguiente disolución de cualquier sistema de

${ }^{8}$ Ubicado en: https://www.un.org/development/desa/dspd/international-yearof-the-family.htm

${ }^{9}$ Ubicado en:

https://www.undp.org/content/undp/es/home/sdgoverview/mdg goals.html normas, tiene un tratamiento prioritario en las actividades de la ONU, la UE.

La política orientada hacia el bien público debería hacer que las familias fuertes fueran una prioridad de la política social. Sin embargo, las normas sexuales se han liberalizado al servicio de las pequeñas minorías, arrebatando así a la familia los valores que la hacen posible. (Gabriele Kuby,2017)

Todo esto favorece y promueve la presencia de la bioética desde su rama: la biofamilia. Desde un enfoque de esta perspectiva se contribuye de alguna manera a proteger a la familia de la demolición, a la que está expuesta, desde su concepto.

\section{Conclusiones}

> La ética no ha perdido interés en nuestra época a pesar de vivir en una sociedad posmoral, como se ha descrito. Y, surge su necesidad debido a la misma complejidad de los dilemas que se presentan a partir de las diversas ideologías que han suplantado a los valores humanistas.

$>$ El término biofamilia surge como una necesidad, es la ética aplicada a la familia y bajo este neologismo se considera tanto al ser humano corpóreo como su pleno desarrollo humano e integral al interior de la familia.

> La familia es considerada coma la célula básica de la sociedad de tal modo que la biofamilia, como visión integral de cada uno de los miembros la familia, forma parte de la sociedad por su interacción inmediata con esta. 
De esta manera uU Urge trabajar en un enfoque de biofamilia que permita aportar soluciones a las reales y verdaderas necesidades de la familia.

- Los organismos supranacionales han desvirtuado el concepto de familia como unidad y abren el concepto a la realidad subjetiva que vive_cada uno de sus miembros, imposibilitando la cohesión en el núcleo bajonúcleo bajo un esquema de desarrollo humano integral. Entre estos organismo supranacionales, la ONU, a través de sus grandes conferencias, generan compromisos en los países participantes; que sin ser vinculantes, exigen que la problemática de la familia propia del país se aborde desde estos compromisos, generando soluciones globales, desconociendo la identidad de cada cultura.

- El enfoque de biofamilia ayudaría a delimitar la verdadera esencia de la familia para que no se diluya con los cambios de época y el concepto de familia como primer grupo de vida.

\section{Recomendaciones}

Se recomienda ampliar las investigaciones sobre el concepto y aplicaciones_de biofamilia.

En el desarrollo del presente artículo surgió el concepto del "biopoder", término acuñado por Michael Faucault hace varias décadas. El autor explica sobre los mecanismos de poder sobre la gestión de la vida que podría ser el origen de la intervención de los organismos supranacionales y su nueva definición de familia enfatizando un enfoque de género. Sin embargo, no se tocó, por no estar dentro de los objetivos, pero se convierte en un nuevo punto de investigación.

\section{Bibliografía}

Bunge Mario, Ardila Rubén (2002). Filosofía de la Psicología. Buenos Aires. Editorial: Siglo veintiuno editores.

Camps Victoria (1991). La Imaginación Ética. Barcelona: Editorial Ariel S.A.

Dulkheim, Émile (2007). La división del trabajo social (Carlos G. Posada, trad.). México. Editorial: Colofón S.A. (Obra original publicada en 1893).

Gabriela Brunet (2006). Ética para todos. México D.F. Editorial: Edere.

Gamboa Bernal, Gilberto Alfonso (2014). Biofamilia: campo poco explorado en bioética. Persona y Bioética, 18(2), undefined-undefined. [Fecha de Consulta 01 de Noviembre de 2019]. ISSN: $0123-$ 3122.

Disponible en: https://www.redalyc.org/articulo.oa?i $\underline{d=832 / 83232594001}$

Kuby Gabriele (2017). La revolución sexual global. La destrucción de la libertad en nombre de la libertad. Madrid. Editorial: Didaskalos.

Lipovetzky Gilles (1994). El crepúsculo del deber. La ética indolora de los nuevos tiempos democráticos. Barcelona: Editorial Anagrama.

López-Montaño, L. M. \& Herrera-Saray, G. D. (2014). Epistemología de la ciencia de Familia - Estudios de familia. Revista Latinoamericana de Ciencias Sociales, Niñez y Juventud, 12 (1), pp. 65-76 
Peña Raquel (2019). La familia como fuente generadora de saberes de los fenómenos sociales, desde una visión transcompleja hacia la educación comunitaria (Tesis doctoral).

Posada González, N. L., López Maldonado, N. Y., Cubillos García, T. P., Vásquez Barrios, A., Briceño Gómez, E., Buriticá Bedoya, D. M., Villa Bedoya, A. V., Díaz Amaya, D. M., Trujillo Charry, M., Cárdenas Hoyos, Y. P. \& Almario Castañeda, F. A. (2017, juliodiciembre). Estado del arte de documentos impresos de mediación pedagógica sobre sexualidad para profesores, niños y adolescentes, enviados por el Ministerio de Educación
Nacional a instituciones de educación formal en Colombia (1990-2014). Rev. Cient. Gen. José María Córdova, 15(20), 101-124.

$\mathrm{DOI}:$ http://dx.doi.org/10.21830/19006586.169

Naciones Unidas (2005). Celebración del décimo aniversario del Año Internacional de la Familia. A/RES/59/147. Ubicado en https://www.un.org/development/desa/ds $\mathrm{pd}$ /international-year-of-the-family.html

Naciones Unidas (1948). Declaración Universal de Derechos Humanos. Ubicado en https://www.undp.org/content/undp/es/h ome/sdgoverview/mdg_goals.html 